\title{
«L'amère mort» di Emma Bovary: intertestualità e metafora critica in Flaubert
}

Daniela De Agostini

\section{(2) OpenEdition}

1 Journals

\section{Edizione digitale}

URL: http://journals.openedition.org/studifrancesi/7803

DOI: $10.4000 /$ studifrancesi.7803

ISSN: 2421-5856

\section{Editore}

Rosenberg \& Sellier

\section{Edizione cartacea}

Data di pubblicazione: 1 juillet 2009

Paginazione: 341-350

ISSN: 0039-2944

\section{Notizia bibliografica digitale}

Daniela De Agostini, «L'amère mort» di Emma Bovary: intertestualità e metafora critica in Flaubert», Studi Francesi [Online], 158 (LIII | II) | 2009, online dal 30 novembre 2015, consultato il 07 janvier 2021. URL: http://journals.openedition.org/studifrancesi/7803 ; DOI: https://doi.org/10.4000/studifrancesi. 7803

\section{(c) (i) $\odot$}

Studi Francesi è distribuita con Licenza Creative Commons Attribuzione - Non commerciale - Non opere derivate 4.0 Internazionale. 


\section{«L'amère mort» di Emma Bovary: intertestualità e metafora critica in Flaubert}

Una serie di circostanze, che non eludono la stretta unione nell'opera flaubertiana di realismo, ironia e vena visionaria, ha portato il romanziere a introdurre, a chiusura della seconda parte di Madame Bovary ${ }^{1}$, la rappresentazione dell'opera di Donizetti, Lucia di Lammermoor ${ }^{2}$, in versione francese, al Théâtre des Arts di Rouen. Questo episodio, sintesi di tutte le forme d'arte, suono, sensazione e concetto, che non solo agevola lo svolgimento della trama, ma getta luce, retrospettivamente e potenzialmente, sul carattere della protagonista, è l'avvenimento conclusivo e per così dire charnière dell'esistenza di Emma. Non solo perché il racconto dell'interpretazione occupa nel romanzo lo stesso posto del celebre sestetto nella partitura dell'opera, ma anche perché costituisce il finale del secondo atto come il récit conclude la seconda parte del romanzo; in entrambi i casi si tratta di un evento, di un coup de théâtre che fa coincidere perfettamente la finzione con la 'realtà': al ritorno inaspettato di Edgar(d), quando Lucie viene unita in sposa a Bucklaw, corrisponde la riapparizione inattesa di Léon, che non ha cessato di amare romanticamente Emma e che ora vuole possederla. E la follia di Lucie, e la sua morte, si rifletteranno come en abyme nel destino di Madame Bovary, che non esiterà a incontrare il nuovo amante e a dilapidare il proprio patrimonio e quello del marito e che, in un attimo di smarrimento, realizzerà il suicidio che già una prima volta l'aveva tentata.

I ricordi comuni, anzitutto, hanno spinto il romanziere a scegliere proprio l'ascolto della rappresentazione della Lucie de Lammermoor come chiusura della seconda parte del suo romanzo: il romanticismo ${ }^{3}$ di Emma Bovary l'ha portata a lungo a sognare, nella sua adolescenza, insieme a Walter Scott, castellane, manieri, fontane e cavalieri, come si legge nella prima parte del romanzo, quando le letture di Emma tredicenne al convento aprono la sua vicenda e disegnano il suo carattere:

Elle avait lu Paul et Virginie et elle avait rêvée la maisonnette de bambous, le nègre Domingo, le chien Fidèle [...]. Avec Walter Scott, plus tard, elle s'éprit de choses historiques,

(1) Gustave Flaubert, Madame Bovary. Moeurs de province, Paris, Bordas, Classiques Garnier, 1990 (edizione a cura di Claudine Gothot-MERSCH).

(2) Si tratta dell'opera di Donizetti, famosissima e grandiosa opera romantica che non solo focalizza il tema dell'amore senza speranza ma è basata su un romanzo di Walter Scott. Riflette anche la moda e la qualità dell'opera italiana a quell'epoca; Emma si ritrova subito nel suo elemento, in un milieu, il melodramma italiano, in cui è a suo agio: felice, in mezzo alla folla, prende posto nel palco "avec une désinvolture de duchesse" (op. cit., p. 227). Cfr. Ugo Piscopo, Le thème de l'Italie dans "Madame Bovary", «Europe», n. 577, mai 1977, p. 170. Si vedano anche GianANDREA GAVAZZENI,
Gaetano Donizetti, Milano, Fratelli Bocca Editori, 1937, AA.VV., Gaetano Donizetti, Milano, 1983, e Tullia FRANZI, La Lucia di Lammermoor nella letteratura romantica, «Rivista di Bergamo», 16, n. 10, ottobre 1937.

(3) Perciò la protagonista flaubertiana "desidera una vita diversa senza saperla neppure concepire, vede amore, lusso, squisitezza dove ci sono soltanto fatuità e squallore, vuol vivere come un'eroina romantica senza saper intravedere altra evasione che l'adulterio e, non riuscendo a percepire la propria tragedia se non come crollo dei suoi labili sogni, naufraga in un abisso meschino di bugie e di debiti [...]" (Giovanni Bogliolo, Flaubert, prima e dopo, Torino, Aragno, 2007, pp. 34-35). 
rêva bahuts, salle des gardes et ménestrels. Elle aurait voulu vivre dans quelque vieux manoir, comme ces châtelaines au long corsage qui, sous le trèfle des ogives, passaient leurs jours, le coude sur la pierre et le menton dans la main, à regarder venir du fond de la campagne un cavalier à plume blanche sur un cheval noir ${ }^{4}$,

e Gustave Flaubert ha certamente assistito per la prima volta alla celebre opera di Donizetti a diciotto anni, quando nel 1839 al Théâtre de la Renaissance si recitava una Lucie de Lammermoor abbreviata nella trama e nella partitura musicale, nella versione francese di Gustave Vaëz e Alphonse Royer ${ }^{5}$, con la collaborazione dello stesso compositore. Un anno prima dell'inizio della redazione del suo capolavoro, nel 1851, la stessa opera veniva rappresentata a Parigi, ed egli, già adulto, al ritorno dal viaggio in Oriente compiuto con l'amico Maxime Du Camp, proprio mentre si accingeva alla composizione della Bovary, si era recato a vederla ${ }^{6}$; in quello stesso anno veniva pubblicata l'opera di Henry Murger, Scènes de la vie de Bohème, il cui protagonista si chiamava Rodolphe, il nome del primo amante di Emma.

La decisione infine di far coincidere l'apprendistato della sua eroina nelle letture che l'hanno formata al convento con la fine dei suoi cicli amorosi, dei suoi adulteri come speranza e illusione di felicità e d'amore dopo le esperienze di un matrimonio deludente, non ha fatto esitare Flaubert che, con Emma Bovary, ha voluto rappresentare in letteratura una delle più importanti demistificazioni del mito dell'amore romantico, dell'assolutismo della passione amorosa che proprio con le letture nasce e si accresce, nutrendosi di esse. (Del resto, anche Walter Scott, nel suo chef-d'œuvre, fa di Lucia una grande lettrice: «Lucia Ashton si trovò spersa nei labirinti dell'immaginazione, pericolosissimi, per le persone giovani e sensibili. [...] una fanciulla di tendenze romantiche, che si deliziava delle storie d'amore e di fantasia al punto da identificarsi con le leggendarie eroine le cui avventure, in mancanza di letture migliori, le avevano riempito la mente» ${ }^{7}$ ). Non da ultima circostanza, la duplice omofonia del titolo dell'opera, Lucie de Lammermoor, con 'l'amara morte' di Emma, e quella del nome del protagonista della finzione narrativa e musicale, Edgar(d), con il cognome fittizio dell'attore che recita l'opera, Lagardy, quasi ad affermare un'identità (Edgar di Ravenswood e Edgar Lagardy), aveva certamente colpito Flaubert quando aveva pensato a quest'ultimo, ritrovando anche nel nome dell'attore che interpretava Edgar(d) il proprio, Gustave. Gustave Roger è infatti uno dei possibili Lagardy ${ }^{8}$, 38.

(4) Gustave Flaubert, Madame Bovary, cit., p.

(5) La celebre opera di Donizetti (1797-1848) su libretto italiano di Salvatore Cammarano, ispirata al romanzo di Walter Scott, The Bride of Lammermoor (1819), fu rappresentata per la prima volta al San Carlo di Napoli il 26 settembre 1835 e, in Francia, il 12 dicembre 1837 al Théâtre-Italien di Parigi. Nella versione francese conobbe due rappresentazioni, una il 6 agosto 1839 al Théâtre de la Renaissance, nella Salle Ventadour, e una all'Opéra, il 20 febbraio 1846. Il libretto italiano è suddiviso in due parti, la prima in un atto unico, la seconda in due atti; quello francese in tre atti.

(6) Una menzione abbastanza casuale della performance di Costantinopoli è stata fatta da Flaubert in Voyages, Paris, Les Belles Lettres, 1948, II, p. 349, e in Correspondance, I, Paris, Gallimard, «Bibliothèque de la Pléiade», 1995, p. 704. Si veda anche Jean BRunEau, Les débuts littéraires de Gustave Flaubert. 1831-1845, Paris, Colin, 1962.

(7) Walter SCOTT, La sposa di Lammermoor, tra- duzione italiana di Oriana Previtali, Milano, Rizzoli, 1951 , p. 75 e p. 332. Come fa osservare GraHAM DANIELS (Emma Bovary's Opera - Flaubert, Scott and Donizetti, «French Studies», XXXII, n. 3, July 1978 , p. 287) se Emma Bovary fosse stata un'assidua lettrice dello scrittore scozzese e avesse letto attentamente questo libro, avrebbe riconosciuto 1'errore' di Lucia nella sua identificazione con le letture romantiche.

(8) Il colto, ironico, sostanzialmente modesto e solerte viaggiatore Roger (che sicuramente cantava la parte di Edgardo nello spettacolo del gennaio 1853) è uno degli interpreti della Lucia. Tra gli altri possibili vi sono l'illustre tenore G.-L. Duprez, la cui carriera culminava proprio con l'affidamento del ruolo di primo interprete nella Lucia di Lammermoor a Napoli nel 1835 e a Parigi nel 1849, cui seguiva un tempestivo ritiro allorché la sua voce cominciava ad affievolirsi; e il celebre divo dell'epoca, un uomo 'del Sud' come il Lagardy flaubertiano: Giovanni Mario, conte di Candia, marito di Giulia Grisi, che fuggì dal regno sardo dov'era nato con 
(dalla voce 'robusta', dalla vita fastosa, di umilissime origini, colto e ironico), nato in un sobborgo di Parigi nel 1815 e morto nella capitale francese nel 1883, cui si deve una frase che è prova della sua fedeltà alla scrittura, e che potrebbe appartenere al romanziere: «Amo l'Arte con passione: essa è stata il culto della mia vita, e quando mi accadeva di gioire degli applausi con i quali i miei sforzi venivano ricompensati, ne ero fiero, non per l'umile sacerdote che io ero, ma per la divinità che servivo»?

Anche se la scelta di Flaubert di inserire nella sua opera l'ascolto della Lucie è stata tardiva rispetto alla redazione del romanzo, la sua genesi è prova della volontà di fare assistere Emma proprio a questo melodramma e di stabilire dunque un ulteriore parallelismo tra i diversi capitoli del romanzo. Metafora critica, la rappresentazione dell'opera soddisfa le funzioni tematiche e strutturali del romanzo, come osserva anche Graham Daniels ${ }^{10}$. I brouillons di Madame Bovary scrivono nel plan e nello scénario partiels il nome di Walter Scott ${ }^{11}$ tra le letture di Emma («Walter Scott - pages - tourelles; apprend à chanter - romances. - histoire Walter Scott»; «ce sont des lectures qu'elle fait après son mariage - Walter Scott. - bon chics historiques -»), e nello scénario d'ensemble appare già il nome di Lucie: «Emma ne se rétablit pas. - distraction conseillée par le curé. Charles la mène à Rouen au spectacle. Été. Port - soir - représentation extraordinaire de la Lucie rencontre de Léon. Conversation au balcon du foyer» ${ }^{12}$.

Le tappe della vicenda sono le stesse, negli abbozzi primitivi e nel romanzo: dopo l'abbandono di Rodolphe e il tentativo di suicidio, Emma si è ammalata, la sua convalescenza è lunga, ma sta abbastanza bene da accettare di recarsi a teatro a Rouen («au spectacle», senza meglio definire la rappresentazione, dicevano dapprima gli scénarios; solo più tardi diventa «la représentation extraordinaire de la Lucie», e una nota a margine del manoscritto nella fase di composizione scrive: «torpeur, c'est à Rouen à la musique qu'elle se ranime» ${ }^{13}$ ), come viene suggerito dal farmacista, Homais, e come Charles - l'ironia di Flaubert in questo romanzo è alleata continua della sua demistificazione del romantico - consiglia, sperando in un diversivo che non potrà apportare che una ulteriore cura, quindi un miglioramento delle condizioni di Emma. Charles Bovary non sa che si celebra il tenore Lagardy nel ruolo dell'amante di Lucie, Edgar(d), e non sa soprattutto che la sua fama di seduttore nella sua vita reale insieme agli effetti della musica e al tenero amore recitato in scena, rilanceranno Emma nel suo passato e, sin dall'inizio del suo ascolto, la getteranno in «insaisissables pensées» ${ }^{14}$ :

Elle se retrouvait dans les lectures de sa jeunesse, en plein Walter Scott. Il lui semblait entendre, à travers le brouillard, le son des cornemuses écossaises se répéter dans les bruyères. D'ailleurs, le souvenir du roman facilitant l'intelligence du libretto, elle suivait l'intrigue phrase

una ballerina e, travestito da pescatore (in analogia con Lagardy) si recava a Parigi dove ebbe occasione di esibirsi in importanti salotti e poi sul palcoscenico. Fu mirabile interprete di Edgardo, guadagnò somme enormi che sperperò in una vita lussuosa e morì in povertà nel 1883, tanto che le sue origini meridionali, la sua vita fastosa, il suo temperamento romantico e le sue avventure, possono essere riconosciuti come tratti comuni al fittizio Lagardy. Altri famosi interpreti di Edgardo sono stati G. Rubini (1837) e A. Ricciardi (1839). Si veda Adriana SANTORO, Flaubert è Bovary: ma chi è il vero Lagardy?, «Museo Teatrale alla Scala», VII, n. 25, 1994-1995.

(9) Le carnet d' un ténor, Paris, 1880, citato da Adriana Santoro, op. cit., p. 126.
(10) Cfr., op. cit., p. 300.

(11) Flaubert possedeva le opere dello scrittore scozzese in traduzione (Euvres complètes de Walter Scott, trad. di Defauconpret, Paris, Furne, 1830). Cfr. anche JEAN BrunEaU, op.cit.

(12) Si vedano Claudine Gothot-Mersch, La genèse de "Madame Bovary", Paris, Corti, 1966 e Gustave Flaubert, Madame Bovary. Ébauches et fragments inédits, Paris, Conard, 1936.

(13) Gustave Flaubert, "Madame Bovary", nouvelle version précédée des scénarios inédits, par Jean Pommier et Gabrielle Leleu, Paris, 1949, pp. 29, 89, 94.

(14) Madame Bovary, cit., p. 228. 
à phrase, tandis que d'insaisissables pensées qui lui revenaient, se dispersaient, aussitôt, sous les rafales de la musique ${ }^{15}$.

Dapprima è il suo passato, e le cocenti ferite del suo matrimonio, a riaffiorare nell'ascolto della musica che lei sembra seguire con lo stesso entusiasmo che l'aveva portata a leggere il libro di Walter Scott: ma i ricordi si disperdono, e soltanto le sue reazioni sono trasmesse al lettore, reazioni che la vedono nell'impossibilità di distanziarsi dalla storia raccontata e di leggere senza identificazione, e capace invece di ricordare ogni situazione romantica e ogni dettaglio passionale che la riflettono narcisisticamente. Flaubert ne dava un inventario non privo di ironia nelle pagine sulle letture di Emma:

Ce n'étaient qu'amours, amants, amantes, dames persécutées s'évanouissant dans des pavillons solitaires, postillons qu'on tue à tous les relais, chevaux qu'on crève à toutes les pages, forêts sombres, troubles du cœur, serments, sanglots, larmes et baisers, nacelles au clair de lune, rossignols dans les bosquets, messieurs braves comme des lions, doux comme des agneaux, vertueux comme on ne l'est pas, toujours bien mis, et qui pleurent comme des urnes ${ }^{16}$.

Così, quando si leva il sipario della Lucie de Lammermoor, Emma si ritrova nel libro di Walter Scott:

Mais on entendit trois coups sur la scène; un roucoulement de timbales commença, les instruments de cuivre plaquèrent des accords, et le rideau, se levant, découvrit un paysage. [...] Il lui semblait entendre, à travers le brouillard, le son des cornemuses écossaises se répéter sur les bruyères [...] Elle se laissait aller au bercement des mélodies et se sentait elle-même vibrer de tout son être comme si les archets des violins se fussent promenés sur ses nerfs ${ }^{17}$.

Se è subito percepibile la sua aderenza al personaggio di finzione, non altrettanto immediatamente il lettore, sulla base dei particolari incoerenti e frammentari ${ }^{18}$ menzionati nel testo, può riconoscere gli elementi, anche quelli più semplici, della trama dell'opera che Emma sta ascoltando: non solo perché l'eroina flaubertiana la ricostruisce ricordando le sue letture di Walter Scott («le souvenir du roman facilitant l'intelligence du libretto, elle suivait l'intrigue phrase à phrase» ${ }^{19}$ ), ma perché, in realtà, Flaubert si ispira, come si è detto, alla versione ritmica francese con varianti di Alphonse Royer e Gustave Vaëz, che Donizetti creò appositamente per il Théâtre de la Renaissance di Parigi e la cui partitura fu considerevolemente modificata «principalmente per adeguarsi alle limitate risorse finanziarie nonché artistiche della com-

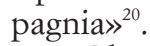

Gli errori e la confusione nei dettagli che Emma fa propri (i costumi, la scenografia, i personaggi, gli strumenti musicali «et les toques de velours, les manteaux, les épées $\left.{ }^{21}\right)$ mostrano infatti come Flaubert, scrivendo questa pagina, avesse l'intenzione di creare un effetto di sovrapposizione tra l'immaginario vissuto della spettatrice e la finzione dell'opera rappresentata. I tocchi di velluto, i mantelli, le spade sono

(15) Ibid.

(16) Ibid., p. 38.

(17) Ibid., p. 228.

(18) «Dal modo in cui Flaubert descrive i frammenti dell'opera come un insieme confuso di scenari, costumi, musica, capiamo che è appunto così che Emma percepisce lo spettacolo: solo che ella accetta la confusione e la frammentarietà come un'esperienza dotata di un ordine significativo. Flaubert descrive, in questo modo, la frammentazione della sua coscienza», osserva e conclude TONY TANNER (L'adulterio nel romanzo, traduzione italiana, Genova, Marietti, 1990, p. 342).

(19) Madame Bovary, cit., p. 228.

(20) W. Ashbrook, Donizetti. Le opere, trad. di L. Della Croce, Torino, EDT, 1987, p. 150.

(21) Madame Bovary, cit., p. 228. 
frammenti delle letture e delle fantasticherie di Emma in convento; la «bourse» che la «jeune femme» getta all'«écuyer vert» («Mais une jeune femme s'avança en jetant une bourse à un écuyer vert» $\gg^{22}$ ) è un particolare che non esiste nel libretto di Cammarano, che si spiega soltanto se si tiene conto della versione francese modificata nel '39, nella quale Lucie entra in scena accompagnata da un nuovo personaggio in cui si fondono il fido Normanno e Alisa, soppressa in questa versione dell'opera. Gilbert, questo il suo nome anche nel romanzo di Walter Scott, «esce di scena prima delle arie di Lucia, e dopo che essa gli ha consegnato un borsellino in cambio della promessa di vigilare su di lei» ${ }^{23}$. Nella stessa versione manca l'«a solo» di arpa che risuona alla comparsa di Lucia, che, nel romanzo di Walter Scott, appare invece preceduta dal suono del liuto:

Nell'attraversare una vasta anticamera gotica, Sir William Ashton udì il suono del liuto di sua figlia. [...] Si fermò, quindi, e rimase ad ascoltare il suono argenteo della voce di Lucia mescolarsi all'accompagnamento, in un'aria antica $[\ldots]^{24}$.

E una borsa «a reticella verde», contenente alcune monete d'oro, è quella che, nel romanzo di Scott, il Signore dei Ravenswood getterà al Capitano Craigengelt quando rifiuterà di lasciare la Scozia per amore di Lucia ${ }^{25}$. Inoltre, se nel libretto di Cammarano la cavatina in sol maggiore con cui Lucia entra in scena («Lucie entama d'un air brave sa cavatine en sol majeur ${ }^{26}$; elle se plaignant d'amour, elle demandait des ailes ${ }^{27}$ ), non «chiedeva delle ali», bensì cantava: «Parmi che a lui d'accanto $\mid$ si schiuda il Ciel per me», in quello della versione francese la protagonista appare per la prima volta intonando l'aria di sortita di Rosmonda d'Inghilterra (1834) e relativa cabaletta, ora diventate «Que n'avons nous des ailes» e «Toi par qui mon cœur rayonne». Nell'immaginario di Emma, e dunque nella scrittura di Flaubert, inizio e fine ancora una volta sembrano confondersi, se si ricorda anche che il primo verso dell'ultima aria cantata da Edgardo, poco prima di morire, recita: «Tu che a Dio spiegasti l'ali». È giusto comunque osservare come il libro di Walter Scott si differenzi profondamente, e soprattutto nel finale, dall'opera. Ma su questo ritorneremo.

Poi, appare Edgar(d)-Lagardy ${ }^{28}$. E sulla sua comparsa, «la taille vigoureuse» e «une de ces pâleurs splendides» che gli donano la maestà marmorea tipica delle «races ardentes du Midi» ${ }^{29}$, infierisce la mordace ironia flaubertiana quando con un ossimoro assimila la sua «admirable nature de charlatan» a un «coiffeur» e a un «toréador» $»^{30}$. (Si osservi che in una bozza del romanzo Flaubert mostra Emma identificarsi con l'opera dopo essersi innamorata dell'attore/cantante e prima di incontrare Léon: «la pièce - gradation - identification - vivre là dedans [que n'avait-elle un Edgar - amoureuse de l'acteur] retour à l'amour» ${ }^{31}$ ). Emma, entusiasta, «se penchait pour le voir, égratignant avec ses ongles le velours de sa loge» ${ }^{32}$ rivivendo attraverso Lucie

(22) Ibid.

(23) W. AsHBroor, op. cit., p. 150. 49.

(24) W. SCOTT, La sposa di Lammermoor, cit., p.

(25) Ibid., p. 87.

(26) La celebre aria di sortita di Lucia, nell'autografo dell'opera, e dunque nella sua versione originale, non è in Sol maggiore ma in $M i$ bemolle e La bemolle. (Cfr. Lucia di Lammermoor, cap. V, p. 146 di W. Ashbrook, op. cit.). La scena del duetto tra Lucia e Enrico è in La e quella della follia in Fa. Queste tonalità originali sono state ripristinate nell’incisione discografica diretta da Jésus López Cobos, con Montserrat Caballé nella parte di Lucia.
(27) Madame Bovary, cit., p. 229.

(28) Di creazione flaubertiana il nome Lagardy non stupisce che sia quasi l'anagramma (eccettuate due lettere) di Edgar, se si tiene conto della trasposizione che in lui effettua Emma da personaggio sulla scena a seduttore senza scrupoli nella vita, e infine all'eroe romantico che era per lei Rodolphe - e, come sarà, poco dopo, lo stesso Léon -. La finzione, sulla scena, è diventata realtà.

(29) Madame Bovary, cit., p. 229.

(30) Ibid.

(31) J. Pommier et G. Leleu, op. cit., p. 100.

(32) Ibid. 
la propria esperienza, ossia le ebbrezze e le angosce che ha conosciuto con Rodolphe. Allorché Edgar(d) e la sposa di Lammermoor, alla fine del primo atto dell'opera, cantano quanto li unisce e li separa, la voce di Emma si confonde con quella della protagonista dell'opera: «La voix de la chanteuse ne lui semblait être que le retentissement de sa conscience, et cette illusion qui la charmait quelque chose même de sa vie»s $^{33}$. Il desiderio e la realtà ancora una volta si fondono per Emma, e il suo autore, in stile indiretto libero, le fa osservare che «personne sur la terre ne l'avait aimée d'un pareil amour $\gg^{34}$, mentre, nella 'stretta' finale, l'addio di Lucie coincide perfettamente con il «cri aigu» ${ }^{35}$, chiaramente erotico, gettato da Emma, che si confonde a sua volta con la vibrazione degli ultimi accordi della musica ${ }^{36}$. L'identificazione è totale, come quella del romanziere con la sua eroina.

Alla fine della prima parte del libretto di Cammarano (o alla fine del primo atto nella versione francese) le parole che si scambiano Edgardo e Lucia (in cui Emma legge la promessa che l'aveva legata a Rodolphe) non accennano però a «fleurs de leur tombe», e neppure a «serments», «exil», «fatalité», «espérances»: a parte l'emblematica parola 'fatalità' ${ }^{37}$, che nell'opera si recita nello scambio degli anelli, quando, prima di separarsi, gli amanti si giurano reciprocamente eterna fedeltà, nell'aria: «Al tuo fato unisco il mio». Quale è descritta da Emma, la scena è più fedele a quella che si legge nel libro di Walter Scott, allorché i due protagonisti si donano una moneta spezzata in due come pegno del loro amore e della loro unione e che Lucia conserva legata al collo con un nastro azzurro ${ }^{38}$.

Si abbassa il sipario, Emma intima a Charles di tacere le sue proteste di incomprensione e, quando il secondo atto dell'opera ha inizio, Lucie avanza «plus pâle que le satin blanc de sa robe» ${ }^{39}$, una coroncina di fiori d'arancio tra i capelli; mentre dapprima ha luogo l'identificazione con il proprio matrimonio poi, con un sorriso che esprime per la prima volta «une pitié dédaigneuse» ${ }^{40}$, Emma sembra distanziarsi dal proprio vissuto:

Elle connaissait à présent la petitesse des passions que l'art exagérait. S'efforçant donc d'en retourner sa pensée, Emma voulait ne plus voir dans cette reproduction de ses douleurs qu'une fantaisie plastique bonne à amuser les yeux, et même elle souriait intérieurement d'une pitié dédaigneuse $[\ldots]^{41}$.

Ella rivede Rodolphe e la propria passione ed esprime la nostalgia, «avant les souillures du mariage et la désillusion de l'adultère», di una felicità non vissuta: «alors la vertu, la tendresse, les voluptés et le devoir se confondant, jamais elle ne serait descendue d'une félicité si haute» ${ }^{42}$, quando entra in scena un nuovo per-

(33) Ibid.

(34) Ibid.

(35) Ibid., p. 230.

(36) $\mathrm{Il}$ «grido acuto» che si lascia sfuggire Emma è un'espressione vagamente simile al grido, vago e prolungato, proveniente dalle colline lontane, che aveva sigillato la conclusione del primo incontro d'amore con Rodolphe: «Alors, elle entendit tout au loin, au delà du bois, sur les autres collines, un cri vague et prolongé, une voix qui se traînait, et elle l'écoutait silencieusement, se mêlant comme une musique aux dernières vibrations de ses nerfs émus» (Ibid., pp. 165-166). Cfr., anche, ADRIANA SANTORO, op. cit., p. 122.

(37) Sotto forma di leggenda, presagio, dichiarazione profetica, apparizione soprannaturale, si noti come il Leitmotiv del fato è predominante nel romanzo di Walter Scott. Nessuna premonizione, ma un uso ironico del cliché appare invece in Flaubert. Si vedano gli scarti del cavallo di Charles Bovary quando si avvicina alla fattoria dei Bertaux, e quelli del cavallo di Edgar Ravenswood allorché giunge alla fonte della leggendaria cattiva reputazione della sua famiglia «come se qualche motivo di terrore si fosse improvvisamente presentato» (WALTER SCOTT, op. cit., p. 188).

(38) Walter SCOTT, La sposa di Lammermoor, cit., pp. 224-232.

(39) Madame Bovary, cit., p. 230.

(40) Ibid., p. 231.

(41) Ibid.

(42) Ibid., p. 230. 
sonaggio: «au fond du théâtre, sous la portière de velours, un homme apparut en manteau noir» ${ }^{43}$.

Riappare Edgar-Lagardy ${ }^{44}$, il cui amore oltraggiato, «intarissable», si riversa sulla folla della platea e il drammatico sestetto si dispiega con l'espressione dei sentimenti di collera, vendetta, gelosia, terrore, compassione e stupore ${ }^{45}$, mentre Emma sembra rinnegare il distanziamento operato poco prima e rivivere la fatalità del proprio vissuto:

Toutes ses velléités de dénigrement s'évanouissaient sous la poésie du rôle qui l'envahissait, et, entraînée vers l'homme par l'illusion du personnage, elle tâcha de se figurer sa vie, cette vie retentissante, extraordinaire, splendide, et qu'elle aurait pu mener cependant, si le hasard l'avait voulu. Ils se seraient connus, ils se seraient aimés!46

Rafforzata dalla omofonia del nome - Edgar/Lagardy - l'identità del personaggio e dell'uomo vanifica ogni velleità di distanziamento da parte di Emma che non solo si lascia andare a sogni di fuga ( «Enlève-moi, emmène-moi, partons! À toi, à toi! Toutes mes ardeurs et tous mes rêves! $\gg^{47}$ ), ma cade vittima dell'isteria tipica di chi ha sofferto una repressione sessuale e una condizione di restrittività sociale ${ }^{48}$. Il sipario si abbassa e quando si rialza nella loge c'è anche Léon, tornato a Rouen dopo tre anni di assenza. Mentre si sta per celebrare il grande finale dell'opera con la scena della follia (alla quale Emma non vuole assistere), lei già non ascolta più, se non nella distanza («dans l'éloignement»), il duetto in re maggiore tra Ashton e il suo valletto, perché sono Léon e il presente che lui le propone, insieme al ricordo del passato, a colmare le sue fantasticherie: «elle se rappelait [...] tout ce pauvre amour si calme et si long, si discret, si tendre, et qu'elle n'avait oublié cependant. Pourquoi donc revenait-il? Quelle combinaison d'aventures le replaçait dans sa vie?» ${ }^{49}$.

La finzione, sulla scena, è diventata realtà: Emma, a teatro, ha trovato la rappresentazione di ciò che aveva cercato nella vita, e cioè un ruolo romantico o poetico nel quale identificarsi che, una volta rimosso Rodolphe, non stenta a riflettersi in Léon. Come en abyme, il duplice finale, del romanzo e dell'opera, si rispecchia con un naturale effetto ironico: il sestetto giunge alla fine del secondo atto, questa scena conclude la seconda parte del romanzo, e la funzione è la medesima. Edgar(d), che ama Lucie, ritorna immediatamente dopo la sua unione con Bucklaw; e Léon, che in segreto ama Emma, è sul punto di riapparire quando la descrizione della rappresentazione viene interrotta prima della follia di Lucie. I risultati sono in entrambi i casi tragici: nell'opera Lucie impazzisce e uccide Arthur prima di morire, nel romanzo Emma diventa l'amante di Léon e poi si uccide («la folie la prenait», e «elle ne souffrait que de son amour», dice la voce del narratore mentre «elle sentait son âme l'abandonner par ce souvenir, comme les blessés, en agonisant, sentent l'existence qui s'en va par leur plaie qui saigne» ${ }^{50}$ ).

Vi sono alcune differenze tra la trama del romanzo di Walter Scott e l'opera, ma le affinità sono capitali. In entrambi Lucia è sposata a un uomo che non ama; e nella

(43) Ibid., p. 231.

(44) Nel libretto di Cammarano Edgardo giunge «ravvolto in gran mantello da viaggio». E Rodolphe, nei suoi appuntamenti notturni, in fondo al giardino, sotto il pergolato, avvolgeva Emma nel suo «grand manteau» (ibid., p. 173).

(45) Il sestetto in musica designa un brano scritto per sei voci o sei strumenti: qui (I, V atto della seconda parte; ovvero del secondo atto) vede riuniti Edgardo, Enrico, Arturo, Raimondo, Lucia, Alisa, e diventò subito famosissimo.

(46) Madame Bovary, cit., 231.

(47) Ibid., p. 232.

(48) Cfr. John R. Williams, Emma Bovary and the Bride of Lammermoor, «Nineteenth-Century French Studies», 20, $\mathrm{n}^{\circ} 3 \& 4$, Spring-Summer 1992 , p. 355.

(49) Madame Bovary, cit., p. 233.

(50) Ibid., p. 319. 
camera nuziale si consuma la sua follia e il suo gesto omicida. Nell'opera compare sulla scena con in mano la spada con cui ha ucciso il marito, prima di morire a sua volta, mentre nel libro è ritrovata nascosta in un canto della stanza, insensibile e muta, caduta in un coma da cui non si riprende dopo aver ferito Arthur Bucklaw; se nella conclusione dell'opera, Edgar si uccide pugnalandosi, venuto a sapere della morte dell'amata, nel romanzo di Scott egli accetta la sfida a duello fattagli dal fratello di Lucy, e muore durante questo incontro, inghiottito dalle sabbie mobili (dove la leggenda aveva predetto che sarebbe morto l'ultimo dei Ravenswood) una volta fuggito dopo il funerale dell'amata, già predetto dalla nutrice. I librettisti dell'opera sacrificano anche la dispotica e intrigante presenza della madre, il lato Macbeth del romanzo, cioè Lady Ashton, e, come si è detto, le diverse comparse della vecchia strega Alsie Gourlay, e si concentrano sulla vicenda del primo amore destinato a spegnersi per l'ostilità dei famigliari. Anche la pagina flaubertiana comporta alcune varianti, nonché l'erronea lettura di Charles e Emma. Se si è nella prima scena del primo atto della seconda parte dell'opera (ovvero nella prima scena del secondo atto), Normanno, capo degli armigeri di Ravenswood, porge a Lord Enrico Ashton, fratello di Lucia, il «simulato foglio» in cui Lucia leggerà il tradimento dell'abbandono di Edgardo: non vi è dunque nessun «faux anneau de fiançailles» come Charles afferma, né un Gilbert che «expose à son maître Ashton ses abominables manœuvres» ${ }^{51}$ (Gilbert era, se mai, il nome del bottaio Girder di The Bride of Lammermoor). Questo personaggio compare invece nella versione francese di Gustave Royer e Alphonse Vaëz: Gilbert, che nel sestetto prende il posto di Alisa, in questa versione rivela la sua falsità nel recitativo, del tutto nuovo, che precede, sempre nel primo atto, il duetto tra Lucie e Henri. È chiaro che mentre Charles non è capace di identificare il rango, i ruoli e le situazioni dei personaggi, non distingue un Lord da un amante, e confonde passione e persecuzione, Emma (anche se è la voce del romanziere che commenta la scena rappresentata) continua e continuerà ad attuare una identificazione totale con l'eroina dell'opera e con la vicenda narrata.

Una 'confusione' di dettagli si rileva anche nella scena inaugurale del terzo atto, quando il sipario si risolleva e Emma rivive nella distanza il grande duetto in re maggiore poiché Léon è giunto e le rêveries del passato la riafferrano: l'opera italiana vede dapprima il canto festoso dei convitati giunti per le nozze, poi il sopraggiungere di Raimondo che, con il récit del «funesto avvenimento», anticipa l'arrivo di Lucia, «le chiome scarmigliate e il volto coperto da uno squallore di morte», come si legge nel recitativo. Nella partitura francese tuttavia, il terzo atto, alquanto modificato e rielaborato per ridurre il numero delle scene, come richiedeva il fondo economico della compagnia del teatro della Renaissance, ha inizio con il coro nuziale ed è seguito subito dall'arrivo di Edgard in casa di Ashton, al quale egli lancia una sfida. Il duetto tenore-baritono, che nel romanzo flaubertiano Emma accosta come 'in lontananza' al coro dei convitati, e che confonde con «la scène d'Ashton et son valet $»^{52}$, con cui si dischiude il terzo atto dell'opera, è seguito da una ripetizione del coro nuziale prima del sopraggiungere di Raimondo con la terribile notizia del suicidio, che è annunciata non da un'aria ma da un recitativo di otto battute. Una volta di più nel pensiero di Emma Bovary presente e passato, della vita e dell'opera, si confondono in un unico atto.

Rimane, ma è esclusa dal romanzo, la scena della follia («Mais la scène de la folie n'intéressait point Emma, et le jeu de la chanteuse lui parut exagéré» ${ }^{53}$ ). Il lettore, e con lui Charles, ne è privato, come è privato dunque del terzo atto, quello della pazzia 
di Lucia e della sua morte. Resta in sospeso il levarsi del sipario sulla terza parte del romanzo flaubertiano, che è nel contempo la terza fase della vita di Emma, con il suo terzo uomo, Léon, dopo il marito e dopo Rodolphe (e la rappresentazione della Lucie, a Rouen, è la terza cerimonia pubblica del romanzo, che ha palesi caratteristiche in comune con la festa da ballo alla Vaubeyssard e con i Comizi agricoli). Sfera fisica, teatrale e lessicale convergeranno infine nell'entrata di Emma nell' atto' da cui non uscirà più, e l'episodio della follia, qui non presente, segna dunque il momento in cui Emma si inoltra in una teatralizzazione confusa della sua stessa esistenza, teatralizzazione che le sarà fatale. Nel romanzo resta l'aria di Edgar, canticchiata («fredonnant ou braillant à plein gosier ${ }^{54}$, osserva la voce del narratore) dagli spettatori che escono dallo spettacolo: «O bell'alma innamorata» («O bel ange, ma Lucie!»), il cui primo verso recita, appunto, e come viene detto all'inizio nel testo, «Tu che a Dio spiegasti l'ali»; e rimane Léon che, esibendo il suo sapere sui tenori (tutti cantanti della troupe italiana), interpreti dell'opera di Donizetti nella rappresentazione del 1837, denigra Lagardy e insieme a lui anche Rodolphe e il possibile amante sognato da Emma: «Il avait vu Tamburini, Rubini, Persiani, Grisi; et, à côté d'eux, Lagardy, malgré ses grands éclats, ne valait rien» ${ }^{55}$, ci dice il discorso indiretto libero flaubertiano.

$$
* * *
$$

L'eliminazione dell'ascolto della scena finale dell'opera ${ }^{56}$ è però anche segno di una ulteriore distanza - se pure mescolata alle numerose affinità - tra l'opera di Donizetti e il romanzo di Flaubert. Come nel libro di Walter Scott, così nella Lucia di Lammermoor non vi è traccia di ironia: l'eroina ama davvero, ricambiata, Edgardo, e il brano musicale che svolge con un lungo monologo la scena della follia indica l'abisso in cui è caduta Lucia che ha perduto il suo sogno d'amore e ha dovuto sposare Arturo. Al contrario per Emma Bovary. Le affinità intertestuali (come il romanzo di Flaubert anche l'originale scottiano era basato su un evento accaduto realmente nella vita) si evidenziano nella trama di entrambe le opere ma l'intento del romanziere francese è profondamente diverso. Emma non sarà una vittima del codice letterario romantico e delle sue convenzioni perché l'ironia flaubertiana vuole colpire soprattutto lei e i suoi sogni, le sue fantasticherie a partire dalle letture in convento ${ }^{57}$, tutte rigorosamente romantiche. In questo stesso episodio, dopo il celebre sestetto, il discorso indiretto libero (tipico dell'arte dello stile di Flaubert) ne illustra il potere e Emma si vede viaggiare, con Edgar-Lagardy, in un effluvio di felicità:

[...] et, entraînée vers l'bomme par l'illusion du personnage, elle tâcha de se figurer sa vie [...] Ils se seraient connus, se seraient aimés! Avec lui, par tous les royaumes de l'Europe, elle

(54) Ibid., p. 234.

(55) Ibid. Tamburini cantava nel ruolo di Enrico (baritono), Rubini (tenore) in quello di Edgardo, Fanny Persiani in quello di Lucia (soprano), una delle sorelle Grisi in quello di Alice (mezzosoprano). La rappresentazione in cui canta Lagardy è perciò successiva e costituisce un punto di riferimento cronologico nel romanzo. Si tratterebbe di quella del 1839 con il tenore Ricciardi (cui Flaubert potrebbe aver assistito nella sua replica il 24 dicembre al Théâtre des Arts di Rouen), o di quella del 1846 con il tenore Duprez, del resto una delle più famose.

(56) Così Graham Daniels: «By rejecting the mad scene and the conclusion of the opera Emma is, more radically even than hitherto, truncating a work of art and rejecting a lesson that art might have taught her: a sense of tragic perspective». ( $O p$. cit., p. 297).

(57) Si osservi che Emma lettrice di romanzi lo è solo dopo il matrimonio nel primo e nel terzo scénarios del romanzo. Flaubert non ha associato, se non circa sei mesi dopo aver dato inizio al romanzo, l'abitudine di Emma alle letture con la sua infanzia e la sua adolescenza. Il nome di Walter Scott appare infatti nelle Notes et plan partiels insieme a quelli di Mme Cottin, Lamartine e alle opere di Goethe, Mme de Staël e Chateaubriand (Werther, Corinne e le Génie du Christianisme). Cfr. Gustave Flaubert, Madame Bovary, nouvelle version précédée des scénarios inédits, cit., p. 4, p. 8; LÉON BOPP, Commentaire sur «Madame Bovary», Paris-Neuchâtel, La Baconnière 1951, p. 71. 
aurait voyagé de capitale en capitale, [...]; puis, chaque soir, au fond d'une loge, derrière la grille à treillis d'or, elle eût recueilli, béante, les expansions de cette âme qui n'aurait chanté que pour elle seule; $[\ldots]$ Mais une folie la saisit: $[\ldots]^{58}$.

Il «genio grammaticale» ${ }^{59}$ flaubertiano coinvolge anche nella sintassi e nel suo contenuto il dissolvimento della realtà che il «livre sur rien ${ }^{60}$ ha inteso rendere; la 'follia' di Lucia può tacere, quella di Emma cominciare.

DANIELA DE AGOSTINI

(58) Madame Bovary, cit., p. 232. Corsivo nostro.

(59) «Et son génie [...] a la forme d'un passé défini, d'un pronom, et d'un participe présent». E Marcel Proust che ha così definito il genio di Flaubert (A ajouter à Flaubert, in Contre SainteBeuve, précédé de Pastiches et mélanges et suivi de Essais et articles, Paris, Gallimard, 1971, «Bibliothèque de la Pléiade», p. 299).

(60) «Ce qui me semble beau, ce que je voudrais faire, c'est un livre sur rien, un livre sans attache extérieure [...], un livre qui n'aurait presque pas de sujet ou du moins où le sujet serait presque invisible, si cela se peut», affermava Flaubert a Louise Colet il 16 gennaio 1852 (Correspondance,
II, cit., p. 31). Cfr. anche il saggio di JEAN RousSET, Madame Bovary ou le livre sur rien, in Forme et signification. Essais sur les structures littéraires de Corneille à Claudel, Paris, Corti, 1963, e di LiOnELlo Sozzi: «Quel rien, infatti, è per noi una parola che allude non tanto all'inconsistenza del contenuto quanto alla nullità, al néant di un reale che è privo di senso. [...] Essenziale e significativa, onnipresente, è infatti, secondo noi, nel romanzo l'idea del nulla [...] Rien si configura ai nostri occhi come parola chiave del testo» ("Ce que je voudrais faire...»: progetto e scrittura in «Madame Bovary», in Il progetto e la scrittura. Le projet et l'écriture, Cagliari-Genova-Torino, Consiglio Nazionale delle Ricerche, 2007, p. 124). 